\title{
Tecnologias não invasivas de cuidado utilizadas por enfermeiras obstétricas: contribuições terapêuticas
}

\author{
Non-invasive care technologies used by obstetric nurses: therapeutics contributions \\ Tecnologías de atención no invasiva utilizadas por las enfermeras obstétricas: contribuciones \\ terapêuticas
}

\author{
Juliana Amaral Prata ${ }^{1}$ (D) \\ Nayara Diniz Pamplona ${ }^{2}$ (D) \\ Jane Márcia Progianti ${ }^{1}$ (]) \\ Ricardo José Oliveira Mouta ${ }^{1}$ (b) \\ Luiza Mara Correia ${ }^{1}$ (1)
}

Adriana Lenho de Figueiredo Pereira ${ }^{1}$ (C)

1. Universidade do Estado do Rio de Janeiro.

Rio de Janeiro, RJ, Brasil.

2. Secretaria Municipal de Saúde. Rio de Janeiro, RJ, Brasil.
Autor correspondente:

Juliana Amaral Prata.

E-mail: juaprata@gmail.com

Recebido em 25/05/2021.

Aprovado em 19/10/2021.

\section{Resumo}

Objetivo: descrever as contribuições terapêuticas da utilização de tecnologias não invasivas de cuidado, oferecidas por enfermeiras obstétricas, durante o trabalho de parto. Método: estudo qualitativo e descritivo, com oito enfermeiras obstétricas da casa de parto do Rio de Janeiro. Os dados foram coletados de setembro a dezembro de 2018, através de entrevistas semiestruturadas, e submetidos à técnica de análise temática. Resultados: para aliviar a dor e promover relaxamento, recorrem ao estímulo à participação do acompanhante e à respiração consciente, à aplicação da massagem, à promoção do ambiente acolhedor e ao uso da água morna e dos óleos essenciais. Para ativar o trabalho de parto, auxiliar na descida da apresentação e correção do posicionamento fetal, incentivam posicionamentos verticalizados e movimentos corporais, com alguns instrumentos. Conclusões e implicações para a prática: tecnologias não invasivas de cuidado possuem contribuições terapêuticas e conformam um cuidado desmedicalizado, respeitoso e centrado na mulher, que promove a autonomia feminina.

Palavras-chave: Cuidados de Enfermagem; Enfermagem Obstétrica; Humanização da Assistência; Gestantes; Tecnologia Biomédica.

\begin{abstract}
Objective: to describe the therapeutics contributions of the use of non-invasive care technologies offered by obstetric nurses during labor. Method: a qualitative and descriptive study, with eight obstetric nurses from a birthing center in Rio de Janeiro. Data were collected from September to December 2018, through semi-structured interviews, and subjected to thematic analysis technique. Results: to relieve pain and promote relaxation, they resort to stimulating the companion's participation and conscious breathing, the application of massage, the promotion of a supportive environment and the use of warm water and essential oils. To activate labor, assist in descending the presentation and correction of fetal positioning, they encourage vertical positioning and body movements, with some instruments. Conclusions and implications for practice: non-invasive care technologies have therapeutic contributions and form a not medicalized, respectful and women-centered care that promotes female autonomy.
\end{abstract}

Keywords: Humanization of Assistance; Nursing Care; Obstetric Nursing; Pregnant Women; Biomedical Technology.

\section{REsumen}

Objetivo: describir las contribuciones terapéuticas de la utilización de tecnologías de atención no invasivas que ofrecen las enfermeras obstétricas durante el trabajo de parto. Método: estudio cualitativo y descriptivo, con ocho enfermeras obstétricas de una casa de partos de Río de Janeiro. Los datos fueron recolectados de septiembre a diciembre de 2018, a través de entrevistas semiestructuradas, y sometidos a la técnica de análisis temático. Resultados: para aliviar el dolor y favorecer la relajación, se recurre a fomentar la participación del acompañante y la respiración consciente, la aplicación de masajes, la promoción de un ambiente acogedor y el uso de agua tibia y aceites esenciales. Para activar el trabajo de parto, facilitan en el descenso de la presentación y corrección del posicionamiento fetal, fomentan las posiciones verticales y los movimientos corporales, con algunos instrumentos. Conclusiones e implicaciones para la práctica: las tecnologías de atención no invasiva tienen aportes terapéuticos y conforman un cuidado desmedicalizado, respetuoso y centrado en la mujer que promueve la autonomía femenina.

Palabras clave: Atención de Enfermería; Enfermería Obstétrica; Humanización de la Atención; Mujeres Embarazadas; Tecnología Biomédica. 


\section{INTRODUÇÃO}

O modelo obstétrico brasileiro se caracteriza pela medicalização que, historicamente, vem apresentando resultados maternos e perinatais desfavoráveis. Nesse contexto, destacam-se iniciativas de qualificação e humanização da assistência ao parto, as quais impulsionam a redução de intervenções desnecessárias e o incentivo ao uso de tecnologias apropriadas e a atuação da enfermagem obstétrica ${ }^{1-3}$.

A inserção das enfermeiras obstétricas no processo de parturição se associa com cuidados desenvolvidos na perspectiva humanística, que promovem autonomia e bemestar, com satisfação das parturientes e desfechos positivos em comparação com o modelo medicalizado. Ademais, verifica-se a ampliação do acesso das mulheres às práticas consideradas benéficas, como presença do acompanhante, oferta de métodos não farmacológicos para alívio da dor, liberdade de movimentos e alimentação ${ }^{2-6}$.

Para a enfermagem obstétrica, esse modo de cuidar se baseia na concepção de desmedicalização, compreendendo que os fenômenos da vida e o processo saúde-doença requerem abordagens que ultrapassam unicamente a clínica-biomédica, admitindo que estratégias de cuidado devem conviver com a autonomia e o direito de escolha da mulher. Sob esta ótica, elas oferecem diferentes tecnologias não invasivas de cuidado de enfermagem (TNICE), definidas como saberes estruturados que se materializam em ações que a enfermeira desenvolve de forma compartilhada com as parturientes, para favorecer a parturição com o mínimo de intervenção e invasão sobre o corpo, estimular seu protagonismo e promover a vivência prazerosa do parto ${ }^{2,4,7}$.

Frente ao exposto, o presente artigo objetivou descrever as contribuições terapêuticas da utilização de tecnologias não invasivas de cuidado, oferecidas por enfermeiras obstétricas, durante o trabalho de parto. Essa pesquisa se faz relevante, pois as publicações científicas comumente se referem às TNICE como métodos não farmacológicos para o alívio da dor; entretanto, acredita-se que a intencionalidade do seu oferecimento durante a parturição ultrapassa o objetivo de minimizar a sensação dolorosa, visto que as práticas dessas especialistas apresentam diversos benefícios e, por isso, são mundialmente reconhecidas como estratégicas para impulsionar a mudança do modelo assistencial obstétrico ${ }^{2,3,5,7}$.

\section{MÉTODO}

Estudo descritivo e qualitativo, com oito enfermeiras obstétricas da Casa de Parto da Secretaria Municipal de Saúde do Rio de Janeiro (SMS/RJ). Foram incluídas aquelas que atuam nos cuidados diretos às mulheres, excluindo-se as que possuem menos de quatro anos na instituição. Assim, das 15 especialistas da casa de parto, quatro foram excluídas pelo tempo de serviço, duas, por licença saúde e maternidade durante a coleta de dados, e uma se recusou a participar.

A coleta dos dados aconteceu de setembro a dezembro de 2018, através de entrevistas semiestruturadas com duração média de 60 minutos, baseadas no seguinte roteiro: você utiliza tecnologias não invasivas de cuidado de enfermagem com as mulheres durante o processo de parturição? Quais tecnologias você utiliza e com quais objetivos? Quais são as contribuições do uso dessas tecnologias para as mulheres?

A captação das participantes iniciou com uma abordagem presencial durante o turno laboral, para uma breve apresentação da pesquisa, seguida do convite à participação. Em caso de aceite, solicitou-se um contato telefônico para o posterior agendamento da entrevista individual.

As entrevistas aconteceram na instituição, antes ou após o horário de trabalho, em ambiente de escolha da participante e assegurando sua privacidade. Foram realizadas, gravadas digitalmente e transcritas por uma das autoras, residente em enfermagem obstétrica na época da coleta dos dados, a qual foi previamente treinada para esta etapa da pesquisa de campo.

As transcrições foram feitas ao término das entrevistas, o que permitiu vislumbrar o momento de redundância dos discursos, indicando a saturação dos dados e determinando o encerramento da coleta ${ }^{8}$. Destaca-se que não houve perdas no decorrer da pesquisa e que foi realizada uma entrevista-piloto, incluída no estudo por não apontar a necessidade de alterações no instrumento.

O material das entrevistas foi submetido à análise temática ${ }^{8}$. Este processo iniciou com a organização e sistematização das entrevistas, seguidas da classificação dos dados e do agrupamento temático, finalizando com a elaboração de sínteses interpretativas que culminaram em duas categorias: Contribuições objetivas do uso das TNICE para as mulheres e Contribuições subjetivas do uso das TNICE para as mulheres.

O estudo foi aprovado pelos Comitês de Ética em Pesquisa da Universidade do Estado do Rio de Janeiro e da SMS/RJ, sob os Pareceres 2.665.608 e 2.883.593. Para preservar o anonimato das participantes, adotou-se a letra $\mathrm{E}$, referente ao termo entrevistada, seguido de algarismos, representando a ordem de realização da entrevista.

\section{RESULTADOS}

As participantes são do sexo feminino, das quais cinco se encontram na faixa etária de 30 anos e três possuem mais de 45 anos. Em relação ao tempo de atuação na assistência ao parto e nascimento, três enfermeiras obstétricas possuem entre 18 e 28 anos, dos quais 14 são na casa de parto, e cinco especialistas possuem entre 5 e 7 anos, sendo 4 na instituição cenário deste estudo.

\section{Contribuições objetivas do uso das TNICE para as mulheres}

Na percepção das participantes, o uso das TNICE contribui para promover relaxamento e conforto, aliviar a sensação dolorosa, auxiliar na descida da apresentação e na correção do posicionamento fetal, bem como ativar o trabalho de parto. 
Se o bebê está alto e tem uma boa dilatação, uso a banqueta. Se a mulher está muito desconfortável, uso a massagem e a banheira, porque o seu uso proporciona alívio das dores da contração. (E1)

Utilizo muito a penumbra, os aromas, oferecidos de acordo com a vontade da mulher. Música para relaxar. Às vezes, vai precisar de rebozo... quando o bebê está assinclítico ou em uma variedade de posição não favorável, mudar ela de posição ou com o spinning babies! (E2, E6, E7)

A grande maioria ama a penumbra por conta do ambiente mais acolhedor e relaxante! [...] o cavalinho, a bola, o rebozo para auxiliar na descida do bebê quando a dilatação está avançada. (E3, E7)

O acompanhante é muito importante! Quando ela está em afinidade com quem a acompanha no trabalho de parto, costuma fluir muito bem! Ela fica muito mais empoderada! (E4)

A massagem para aliviar as dores na lombar. [...] na banheira, elas relatam melhora da dor. Os aromas entram como relaxantes ou ativadores do trabalho de parto! O escalda pés usamos para a ativação do trabalho de parto. (E5, E6)

Temos exercícios para ajeitar melhor o bebê na pelve! A bola para fortalecer a musculatura e ampliar o espaço na pelve! (E8, E7)

\section{Contribuições subjetivas do uso das TNICE para as mulheres}

As participantes reconhecem que a utilização das TNICE se traduz no oferecimento de cuidados de enfermagem sensíveis, respeitosos e individualizados, que favorecem o protagonismo da mulher, o exercício de seus direitos, liberdade de decisão e de escolha, acesso às informações e satisfação com a parturição.

Se pensarmos que o conceito de humanização é a mulher ser protagonista, viver o momento de forma ativa... garantir a ela esse direito de escolha é a principal contribuição das tecnologias. (E4, E2)

Garantir o direito ao conhecimento e de escolha à mulher! Aqui, elas têm autonomia nas decisões e, mesmo quando é necessária uma intervenção, elas são avisadas do porquê, para quê... têm o conhecimento para saber quais tecnologias querem usar, quais não Ihe agradam, para quê elas servem. O protagonismo que todo o conhecimento traz para elas é uma das contribuições! É mostrar para elas que nós somos meros expectadores, que podem ou não precisar intervir! (E5, E3)

É o cuidado diferenciado e único! Através dessas tecnologias, a gente consegue oferecer tudo o que a mulher precisa nesse processo: conhecimento, empoderamento, liberdade de escolha, poder de decisão. (E6, E1)
Essas tecnologias resgatam o protagonismo delas que, às vezes, se perdeu ao longo da vida! É um cuidado mais singular! Cada mulher tem uma fisiologia diferente $e$ cada parto se dará de uma maneira! Então, o uso dessas tecnologias será único! [...] E elas sentem isso! Dar a elas a autonomia para viver esse processo! A segurança $e$ satisfação que elas citam! (E7, E1, E2, E8)

\section{DISCUSSÃO}

O processo analítico revelou que as enfermeiras obstétricas utilizam diferentes TNICE com as mulheres durante a parturição na casa de parto, reconhecendo suas contribuições objetivas, que correspondem à relação entre as ações e os resultados concretos; e subjetivas, que decorrem da visão de mundo que as orienta no processo de cuidar, agregando significados às experiências e gerando resultados abstratos.

Com o intuito de promover conforto e relaxamento, as participantes recorrem às seguintes TNICE: incentivo à presença, participação e envolvimento do acompanhante; estímulo à respiração consciente; promoção de ambiente acolhedor; e aplicação de conhecimentos acerca dos óleos essenciais, associados ou não à técnica da massagem. Essas tecnologias se apresentaram nas falas, respectivamente, por meio dos termos "acompanhantes", "respiração", "penumbra", "músicas e incensos" e "aromas ou aromaterapia".

A participação do acompanhante de escolha da mulher no parto é um direito e uma prática obstétrica recomendada, pois se traduz em apoio emocional, conforto físico, suporte informativo, motivação, segurança, confiança, relaxamento e tranquilidade, percepção de protagonismo da parturiente e de vivência positiva do parto. Esta TNICE se relaciona com o aumento de partos espontâneos, redução da duração do trabalho de parto e da ocorrência de complicações, de analgesia, intervenções desnecessárias, cesarianas, partos instrumentais e asfixia neonatal ${ }^{9-12}$.

O estímulo à respiração consciente na parturição é uma técnica que consiste em alternar períodos de relaxamento da musculatura corporal com diferentes padrões respiratórios ${ }^{9,10,12}$. Geralmente utilizada em associação com outras técnicas, age como um mecanismo de controle do sistema nervoso que, através da ativação de processos mentais que tornam as sensações do trabalho de parto mais agradáveis, promove redução dos níveis circulantes de hormônios do estresse e liberação de endorfinas, diminuindo a pressão sanguínea e aumentando os níveis de oxigênio ${ }^{13,14}$.

Apesar da baixa qualidade das evidências científicas disponíveis, a respiração consciente promove equilíbrio emocional, relaxamento, encorajamento, vigor, conforto e bem-estar, diminuindo o nível de ansiedade e auxiliando no enfrentamento das contrações, com redução da duração do trabalho de parto ${ }^{12-14}$.

O oferecimento da música e da penumbra expressa a preocupação das enfermeiras com o ambiente da parturição 
como um fator que influencia no bem-estar das mulheres, visto que o parto envolve um processo de ativação da região primitiva do cérebro para alcançar o equilíbrio hormonal necessário à evolução fisiológica. Por outro lado, a falta de privacidade e de atenção dispensada pelos profissionais, as intervenções desnecessárias, o excesso de luz e ruídos, a ansiedade e o estresse podem interferir negativamente, inibindo o córtex primal e estimulando o neocórtex, região do cérebro responsável pelo raciocínio ${ }^{15}$.

Assim, recomenda-se que o ambiente do parto seja acolhedor e confortável, com baixa luminosidade, sendo um cuidado que contribui para a humanização da assistência, com respeito à fisiologia, à privacidade e à individualidade da mulher, além de transmitir tranquilidade, minimizar a sensação dolorosa, favorecer a concentração, melhorar a percepção corporal e de autonomia da parturiente ${ }^{9,10,15}$

Corroborando para um ambiente agradável, destaca-se a musicoterapia, que é a utilização de músicas para acionar áreas do encéfalo relacionadas às emoções e ao sistema de recompensas. Na parturição, minimiza a ansiedade, o estresse e o medo, aumenta o vigor e o ânimo, diminui o pulso cardíaco e os esforços respiratórios, apresentando resultados positivos sobre a percepção dolorosa ${ }^{13,16}$.

Além da baixa luminosidade e de música ambiente, as participantes também pontuaram o uso de aromas, por meio de incensos ou de óleos, para criar uma atmosfera relaxante. Segundo a terapia tradicional chinesa, a aromaterapia consiste na aplicação de óleos essenciais para recuperar o equilíbrio e a harmonia do organismo visando à promoção da saúde física e mental ${ }^{4,17}$.

Infere-se que os óleos essenciais agem na liberação de encefalinas e endorfinas, as quais possuem efeito analgésico e produzem sensação de bem-estar e relaxamento, com ações sobre os parâmetros fisiológicos e na resposta ao estresse. Por via inalatória, há estimulação de células nervosas olfativas que ativam receptores do sistema límbico, responsáveis pelas emoções, sentimentos e impulsos motivacionais ${ }^{16}$.

Considerando as propriedades terapêuticas de cada óleo essencial e as singularidades de cada mulher, o oferecimento na atenção obstétrica pode ser através da difusão ambiental, da inalação, do uso tópico ou da diluição em água, sendo comum a associação com a massagem ou escalda pés ${ }^{4,16,17}$.

Outra contribuição das TNICE apontada pelas participantes é aliviar a sensação dolorosa por meio: do uso da água morna no banho de imersão, denominada por elas como banheira da aplicação de massagem; do incentivo à deambulação; e do estímulo à respiração consciente, que também é utilizada para promover conforto e bem-estar.

O banho quente ou hidroterapia é uma técnica acessível, de baixo custo e com alta efetividade, que consiste no uso da água em torno de $37^{\circ} \mathrm{C}$ por, no mínimo, 20 minutos, para lentificar a transmissão aferente nociceptiva dos termorreceptores da epiderme e reduzir a atividade simpática, diminuindo os níveis de hormônios neuroendócrinos relacionados ao estresse e elevando os níveis de encefalinas e endorfinas endógenas ${ }^{12,16,18}$

A utilização da água morna durante a parturição promove vasodilatação periférica, relaxamento da musculatura local e aumento da tolerância à dor. Por isso, apresenta benefícios sobre a progressão fisiológica do trabalho de parto, pois, ao melhorar a distribuição do fluxo sanguíneo muscular, diminui o estresse provocado pelas contrações, regula a contratilidade uterina, favorece a dilatação cervical e auxilia na rotação do polo cefálico, sobretudo em apresentações fetais posteriores ${ }^{16,18}$

Sob a forma do banho de aspersão ou de imersão, o uso da água morna é recomendado como um método não farmacológico de alívio da dor durante o trabalho de parto ${ }^{9,10}$. Contudo, seu uso ultrapassa este benefício, sendo uma das TNICE mais demandadas pelas parturientes, pois se associa com a sensação de revigoramento, conforto, relaxamento e bem-estar, percepção de autonomia, participação do acompanhante e satisfação com a parturição ${ }^{4,18}$

A massagem é uma técnica de estimulação sensorial, baseada no toque sistêmico e na manipulação dos tecidos, que ativa os sistemas simpático e parassimpático, reduzindo a secreção de adrenalina e noradrenalina, bem como aumentando a liberação de endorfinas e ocitocina ${ }^{19}$. Seu uso é recomendado para reduzir ansiedade e estresse, promover relaxamento e conforto, aliviar dores e desconfortos, diminuir fadiga muscular $e$ propiciar consciência corporal e equilíbrio emocional ${ }^{12,19}$. Durante a parturição, a aplicação de massagem favorece a evolução fisiológica, reduz a duração do trabalho de parto, melhora as contrações uterinas, fortalece o vínculo com o profissional e se reverte em maior satisfação ${ }^{9,10,16}$.

O incentivo à deambulação se relaciona com o estímulo à liberdade de movimentos das mulheres durante o trabalho de parto. Historicamente, predominavam as posições horizontais, nas quais as vértebras da mulher se mantinham alinhadas entre 0 e 45 graus $^{20}$. Entretanto, as recomendações atuais apontam para o incentivo à livre movimentação, incluindo a deambulação, e a adoção de posicionamentos confortáveis, principalmente verticalizados 9,10

As posições verticais (em pé, sentada em cadeira, banqueta ou cavalinho de parto, ajoelhada, de cócoras ou de quatro apoios) devem ser encorajadas, pois, ao viabilizarem o alinhamento das vértebras em um ângulo igual ou superior à 90 graus e favorecerem a mobilidade pélvica concomitante à ação da gravidade sobre o eixo central do corpo, apresentam benefícios importantes para a progressão fisiológica do parto, com satisfação das mulheres por proporcionarem conforto, participação ativa, maior percepção de respeito e de controle sobre o processo ${ }^{20}$.

A liberdade de movimentos permite a deambulação e a alternância de posições, relacionando-se com melhora da dinâmica uterina, aumento da tolerância à dor, otimização da dilatação cervical e da descida da apresentação fetal, aceleração da fase ativa e redução do trabalho de parto, do uso de analgesia e da ocorrência de cesarianas. Já no período expulsivo, melhora os puxos maternos, a oxigenação fetal e os resultados perineais, 
favorecendo a mobilidade do sacro e a ampliação dos diâmetros pélvicos ânteroposterior e transverso ${ }^{16,20}$.

Com a intencionalidade de auxiliar na descida da apresentação e na correção do posicionamento fetal, as participantes recorrem às seguintes TNICE: incentivo à deambulação, também utilizado para aliviar a dor; aplicação de conhecimentos acerca das propriedades dos óleos essenciais, associados ou não à técnica da massagem; incentivo aos posicionamentos verticalizados, com o uso de bola, banqueta ou cavalinho; e estímulo aos movimentos corporais e pélvicos específicos da mulher, denominado por elas como rebozo e spinning babies.

Quando o incentivo às posições verticalizadas ultrapassa o objetivo de amenizar a dor, as enfermeiras obstétricas utilizam a bola suíça, um instrumento lúdico que desloca o foco de sensações desagradáveis e apresenta ações terapêuticas ${ }^{12,18}$. Na obstetrícia, o uso da bola proporciona a participação ativa da mulher, melhora a circulação uterina e as contrações, auxilia na descida e na progressão fetal, promove conforto, alivia a dor, favorece a progressão do trabalho de parto e está associado à redução do uso de analgesia e ocitócitos, bem como da ocorrência de episiotomia, traumas perineais, partos instrumentais e desfechos cirúrgicos ${ }^{16,18}$.

Esses benefícios advêm do posicionamento verticalizado, que propicia a ação da gravidade e o alinhamento do eixo fetal com a pelve materna, e do relaxamento de músculos perineais, propiciado por exercícios pélvicos ativos de alongamento e mobilidade sobre a bola ${ }^{16,18}$. Em associação com a técnica da massagem, com o incentivo à respiração consciente ou com uso da água morna na fase de dilatação, constatam-se redução da dor, promoção do conforto, menores índices de analgesia peridural e maior ocorrência de parto normal ${ }^{12,18}$.

A banqueta e o cavalinho são instrumentos com benefícios semelhantes ao uso da bola e de posicionamentos verticalizados. O primeiro é um banco baixo em formato de meia lua, que favorece a descida da apresentação fetal no trabalho de parto ou no período expulsivo. Já o segundo é similar à uma cadeira com assento invertido e apoio para os braços, que proporciona a inclinação do tórax para frente e o balanço pélvico, bem como o relaxamento da musculatura lombar durante o trabalho de parto ${ }^{16}$.

Transparecendo nas falas das participantes, como rebozo e spinning babies, o estímulo aos movimentos corporais e pélvicos específicos é uma TNICE que consiste em exercícios de relaxamento e de mobilidade utilizados na gestação e no trabalho de parto, incluindo: caminhada; alongamento de panturrilha; agachamento; mobilização do tipo lunge; abertura de quadril; balanço das pernas; inversão inclinada para frente; elevação da barriga, em posição de quatro apoios; alongamento estático miofascial; e liberação do sacro ${ }^{21,22}$.

O rebozo é uma técnica que utiliza um xale para movimentar a pelve de forma rítmica, suave e controlada, com o intuito de promover relaxamento muscular e dos ligamentos da região lombar e pélvica, ajudar na descida da apresentação fetal ou corrigir casos de assinclitismo, sendo contraindicada diante de desconforto materno, frequência cardíaca fetal não tranquilizante, sangramento vaginal anormal e risco de prolapso de cordão ou descolamento placentário ${ }^{21}$.

O spinning babies é uma abordagem baseada em conhecimentos da anatomia e da fisiologia, para favorecer a parturição com recursos naturais que estimulam a consciência corporal, otimizam o alinhamento das posições materna e fetal, auxiliam na acomodação fetal, promovem relaxamento e alongamento muscular e de ligamentos, aumentam a flexibilidade e a mobilidade da pelve, proporcionando conforto e minimizando a dor ${ }^{22}$.

Apesar da ausência de estudos clínicos, os benefícios do rebozo e do spinning babies estão documentados em relatos de experiência e narrativas de mulheres que revelam resultados positivos sobre a progressão do trabalho de parto, a dinâmica uterina e o alívio da dor, associando-se com sensações corporais agradáveis, percepção de apoio emocional, empoderamento, prazer, bem-estar, cooperação e segurança ${ }^{21}$. Ainda que não estejam mencionadas em recomendações oficiais, entende-se que essas técnicas se aproximam de práticas obstétricas que promovem e protegem o processo fisiológico do parto, como o incentivo à deambulação, à liberdade de movimentos e aos posicionamentos verticalizados ${ }^{9,10}$.

Para ativar o trabalho de parto, além do estímulo aos movimentos corporais e pélvicos e da aplicação de conhecimentos acerca dos óleos essenciais, associados ou não à técnica da massagem, as participantes também recorrem à técnica de imersão dos pés em água morna, como uma TNICE que denominam de "escalda pés".

Esta técnica é indicada para aliviar edemas e desconfortos nos membros inferiores, pois a água morna promove a vasodilatação e favorece a circulação sanguínea. Ainda, ao evocar sensações agradáveis, promove relaxamento e reduz a fadiga e a ansiedade, por aumentar a atividade parassimpática, com liberação de endorfinas acompanhada da diminuição dos níveis de cortisol e adrenalina ${ }^{23}$.

Além das contribuições terapêuticas evidenciadas neste estudo, as falas das participantes também mostram o reconhecimento do protagonismo da mulher na parturição. Tal constatação revela que o uso das TNICE conforma um processo de cuidar respeitoso e centrado na mulher, que possui características desmedicalizadas e apresenta como contribuições subjetivas o desenvolvimento de cuidados de enfermagem individualizados, singulares, sensíveis, educativos e emancipadores, que promovem a autonomia feminina.

Apontada como estratégia para qualificar a atenção obstétrica e melhorar a satisfação das parturientes, a promoção de cuidados maternos respeitosos consiste em uma abordagem à mulher baseada nos direitos humanos fundamentais, que consideram suas necessidades e preferências, bem como proporcionam o acesso equitativo à assistência baseada em evidências científicas ${ }^{2,6}$.

Já o cuidado centrado na mulher é um modelo teórico de práticas na atenção ao parto e nascimento, fortemente associado com a atuação de enfermeiras obstétricas e adotado em diversos países, como EUA, Nova Zelândia, Escócia, Suécia, Islândia e 
África do Sul, que envolve acolhimento, vínculo e comunicação como elementos fundamentais para o estabelecimento de uma relação na qual a mulher é tratada de forma digna e participa ativamente da tomada de decisão sobre sua saúde ${ }^{5,24}$.

Enquanto características observadas nas falas das enfermeiras obstétricas deste estudo, cuidados respeitosos e centrados na mulher perpassam por atitudes, comportamentos e práticas que: proporcionam um ambiente seguro; demonstram gentileza, disponibilidade e segurança; compartilham informações sobre as possibilidades de assistência; garantem o consentimento informado e a continuidade do apoio familiar; propiciam a comunicação clara, eficaz e livre de julgamentos; asseguram privacidade, confidencialidade e dignidade; envolvem a mulher nos processos decisórios; apoiam suas escolhas; respeitam sua cultura, crenças, desejos e subjetividades s,6,9,11,24. $^{\text {. }}$

Assim, nota-se que esses princípios da assistência obstétrica estão alinhados com a concepção de desmedicalização, que pressupõe incorporar premissas do modelo humanístico e saberes de diferentes campos do conhecimento, para compreender a parturição como um fenômeno natural da vida, valorizar o protagonismo feminino, apresentar opções de cuidado e compartilhar as decisões ${ }^{2-5,7}$.

Frente ao exposto, a realização desta pesquisa revelou que o oferecimento das TNICE pelas enfermeiras obstétricas na casa de parto é parte de um cuidar desmedicalizado, respeitoso e centrado na mulher, que se baseia em um processo interacional com intencionalidade, produção de subjetividades e ações negociadas para o alcance de objetivos terapêuticos na parturição. Desse modo, por meio da associação de conhecimentos, técnicas e procedimentos com atitudes e comportamentos dialógicos e sensíveis, o cuidado agrega atributos não invasivos, alcança a integralidade do ser humano, resguarda a fisiologia do corpo feminino e, sobretudo, assegura o exercício de cidadania das mulheres.

\section{CONCLUSÕES E IMPLICAÇÕES PARA A PRÁTICA}

As enfermeiras obstétricas utilizam diferentes TNICE com as mulheres durante o processo de parturição na casa de parto, as quais apresentam indicações específicas, contribuições objetivas, que consubstanciam seu uso terapêutico, e contribuições subjetivas, que perpassam por uma relação de cuidado desmedicalizado, respeitoso e centrado na mulher, com práticas obstétricas alinhadas com os princípios de integralidade, humanização e autonomia.

Como limitação deste estudo, destaca-se a lacuna de conhecimentos sobre as TNICE, visto que a maioria das publicações as abordam como métodos não farmacológicos para alívio da dor, desconsiderando seu potencial subjetivo e transformador para as mulheres. Assim, sugere-se a realização de pesquisas de validação das TNICE, a fim de produzir evidências científicas que relacionem suas contribuições com a satisfação das mulheres, a qualidade e a segurança da atenção obstétrica.
Ademais, ressalta-se o fato de as participantes, predominantemente, referirem às TNICE como instrumentos oriundos do processo industrial e práticas integrativas e complementares. Tal constatação carece de maiores reflexões, pois a adoção deste recurso discursivo pode interferir no reconhecimento social das enfermeiras obstétricas e na autonomia profissional da especialidade.

\section{CONTRIBUIÇÕES DOS AUTORES}

Desenho do estudo. Juliana Amaral Prata. Nayara Diniz Pamplona.

Coleta ou produção dos dados: Nayara Diniz Pamplona.

Análise de dados. Juliana Amaral Prata. Nayara Diniz Pamplona.

Interpretação dos resultados. Juliana Amaral Prata. Nayara Diniz Pamplona. Jane Márcia Progianti. Ricardo José Oliveira Mouta. Luiza Mara Correia. Adriana Lenho de Figueiredo.

Redação e revisão crítica do manuscrito. Juliana Amaral Prata. Nayara Diniz Pamplona. Jane Márcia Progianti. Ricardo José Oliveira Mouta. Luiza Mara Correia. Adriana Lenho de Figueiredo Pereira.

Aprovação da versão final do artigo. Juliana Amaral Prata. Nayara Diniz Pamplona. Jane Márcia Progianti. Ricardo José Oliveira Mouta. Luiza Mara Correia. Adriana Lenho de Figueiredo Pereira.

Responsabilidade por todos os aspectos do conteúdo e a integridade do artigo publicado. Juliana Amaral Prata. Nayara Diniz Pamplona. Jane Márcia Progianti. Ricardo José Oliveira Mouta. Luiza Mara Correia. Adriana Lenho de Figueiredo Pereira.

\section{EDITOR ASSOCIADO}

Stela Maris de Melo Padoin (D)

\section{EDITOR CIENTÍFICO}

\author{
Ivone Evangelista Cabral (D)
}

\section{REFERÊNCIAS}

1. Leal MC, Bittencourt SA, Esteves-Pereira AP, Ayres BVS, Silva LBRAA Thomaz EBAF et al. Avanços na assistência ao parto no Brasil: resultados preliminares de dois estudos avaliativos. Cad Saude Publica. 2019;35(7):e00223018. http://dx.doi.org/10.1590/0102-311x00223018. PMid:31340337.

2. Medeiros RMK, Teixeira RC, Nicolini AB, Alvares AS, Corrêa ACP, Martins DP. Cuidados humanizados: a inserção de enfermeiras obstétricas em um hospital de ensino. Rev Bras Enferm. 2016;69(6):1091-98. http:// dx.doi.org/10.1590/0034-7167-2016-0295. PMid:27925085.

3. de Souza KCR, da Silva TPR, Damasceno AKC, Manzo BF, Souza KV, Filipe MML et al. Coexistence and prevalence of obstetric interventions: an analysis based on the grade of membership. BMC Pregnancy Childbirth. 2021;21(1):618. http://dx. doi.org/10.1186/s12884-02104092-x. PMid:34503471.

4. Duarte MR, Alves VH, Rodrigues DP, De Souza KV, Pereira AV, Pimentel MM. Tecnologias do cuidado na enfermagem obstétrica: contribuição para o parto e nascimento. Cogitare Enferm. 2019;24:e54164. http:// dx.doi.org/10.5380/ce.v24i0.54164.

5. Lundgren I, Berg M, Nilsson C, Olafsdottir OA. Health professionals perceptions of a midwifery model of woman-centred care implemented 
on a hospital labour ward. Women Birth. 2020;33(1):60-9. http://dx.doi. org/10.1016/j.wombi.2019.01.004. PMid:30686654.

6. Shakibazadeh E, Namadian M, Bohren MA, Vogel JP, Rashidian A, Nogueira Pileggi V et al. Respectful care during childbirth in health facilities globally: a qualitative evidence synthesis. BJOG. 2018;125(8):932-42. http://dx.doi.org/10.1111/1471-0528.15015. PMid:29117644.

7. Prata JA, Ares LPM, Vargens OMC, Reis CSC, Pereira ALF, Progianti JM. Non-invasive care technologies: nurses' contributions to the demedicalization of health care in a high-risk maternity hospital. Esc Anna Nery. 2019;23(2):e20180259. http://dx.doi.org/10.1590/21779465-ean-2018-0259.

8. Minayo MCS. O desafio do conhecimento: pesquisa qualitativa em saúde. $11^{\text {a }}$ ed. São Paulo: Hucitec; 2017.

9. World Health Organization. WHO recommendations: intrapartum care for a positive childbirth experience [Internet]. Geneva: WHO; 2018 [citado 2019 fev 8]. Disponível em: https://apps.who.int/iris/bitstream/ handle/10665/260178/9789241550215-eng.pdf?sequence=1

10. Ministério da Saúde (BR). Secretaria de Ciência, Tecnologia e Insumos Estratégicos. Departamento de Gestão e Incorporação de Tecnologias em Saúde. Diretrizes nacionais de assistência ao parto normal: versão resumida [Internet]. Brasília: Ministério da Saúde; 2017 [acesso 2019 fev 8]. Disponível em: http://bvsms.saude.gov.br/bvs/publicacoes/ diretrizes_nacionais_assistencia_parto_normal.pdf

11. Bohren MA, Berger BO, Munthe-Kaas H, Tunçalp Ö. Perceptions and experiences of labour companionship: a qualitative evidence synthesis. Cochrane Database Syst Rev. 2019;3(7):CD012449. http://dx.doi. org/10.1002/14651858.CD012449.pub2. PMid:30883666.

12. Lehugeur D, Strapasson MR, Fronza E. Non-pharmacological management of relief in deliveries assisted by an obstetric nurse. Rev Enferm UFPE. 2017;11(12):4929-37. https://doi.org/10.5205/1981 8963-v11i12a22487p4929-4937-2017.

13. Smith CA, Levett KM, Collins CT, Armour M, Dahlen HG, Suganuma M Relaxation techniques for pain management in labour. Cochrane Database Syst Rev. 2018;3(3):CD009514. http://dx.doi.org/10.1002/14651858. CD009514.pub2. PMid:29589650

14. Cicek S, Basar F. The effects of breathing techniques training on the duration of labor and anxiety levels of pregnant women. Complement Ther
Clin Pract. 2017;29:213-9. http://dx.doi.org/10.1016/j.ctcp.2017.10.006. PMid:29122264.

15. Rodrigues LSP, Shimo AKK. Baixa luminosidade em sala de parto: vivências de enfermeiras obstétricas. Rev Gaúcha Enferm. 2019;40:e20180464. http://dx.doi.org/10.1590/1983-1447.2019.20180464. PMid:31531594.

16. Araújo ASC, Correia AM, Rodrigues DP, Lima LM, Gonçalves SS Viana APS. Non-pharmacological methods in home birth. Rev Enferm UFPE on Line. 2018;12(4):1091-6. https://doi.org/10.5205/1981-8963v12i4a230120p1091-1096-2018.

17. Chen SF, Wang CH, Chan PT, Chiang HW, Hu TM, Tam KW et al. Labour pain control by aromatherapy: A meta-analysis of randomised controlled trials. Women Birth. 2019;32(4):327-35. http://dx.doi.org/10.1016/j. wombi.2018.09.010. PMid:30344029.

18. Henrique AJ, Gabrielloni MC, Cavalcanti ACV, Melo PS, Barbier M. Hidroterapia e bola suíça no trabalho de parto: ensaio clínico randomizado. Acta Paul Enferm. 2016 dez;29(6):686-92. http://dx.doi. org/10.1590/1982-0194201600096.

19. Smith CA, Levett KM, Collins CT, Dahlen HG, Ee CC, Suganuma M Massage, reflexology and other manual methods for pain management in labour. Cochrane Database Syst Rev. 2018;3(3):CD009290. http:// dx.doi.org/10.1002/14651858.CD009290.pub3. PMid:29589380.

20. Gupta JK, Sood A, Hofmeyr GJ, Vogel JP. Position in the second stage of labour for women without epidural anaesthesia. Cochrane Database Syst Rev. 2017;5(5):CD002006. http://dx.doi.org/10.1002/14651858. CD002006.pub4. PMid:28539008.

21. Iversen ML, Midtgaard J, Ekelin M, Hegaard HK. Danish women's experiences of the rebozo technique during labour: A qualitative explorative study. Sex Reprod Healthc. 2017;11:79-85. http://dx.doi. org/10.1016/j.srhc.2016.10.005. PMid:28159133.

22. Tully G. Spinning babies: guia de consulta rápida. Tradução de Luciana Carvalho. São Paulo: Lexema; 2016.

23. Zainiyah Z, Sunsati E, Asrifah A. The effect of warm foot bath with salt of edema under extremity in postpartum preeclampsia. J Midwifery. 2019;4(1):78-84. https://doi.org/10.25077/jom.4.1.78-84.2019.

24. Brady S, Lee N, Gibbons K, Bogossian F. Woman-centred care: an integrative review of the empirical literature. Int J Nurs Stud. 2019;94:10719. http://dx.doi.org/10.1016/j.ijnurstu.2019.01.001. PMid:30951986. 